\title{
Incidencia de los Paradigmas de la Matemática en la Enseñanza y Aprendizaje de la Estadística
}

\section{Incidence of Mathematics Paradigms in the Teaching and Learning of Statistics}

DOI: $10.46932 / \mathrm{sfjdv2n2-094}$

Received in: March 1st, 2021

Accepted in: May 30th, 2021

\author{
Héctor Hevia \\ Doctor of Philosophy \\ Universidad Alberto Hurtado \\ Río claro 12187911204 Peñalolén Santiago, Chile \\ E-mail: hctr.hevia@gmail.com
}

\section{RESUMEN}

Se analizan algunas situaciones que se presentan en el aprendizaje de la Estadística las que podrían ser simplificadas y aprovechadas en mejor manera, si se sustraen ciertos elementos matemáticos prescindibles y se agregan actividades de aprendizaje apropiadas, que faciliten la familiarización de los estudiantes con ciertos aspectos fundamentales del pensamiento estadístico. Dado que estas propuestas utilizan experiencias de la vida real, ellas posibilitan una construcción cognitiva basada en la teoría de Bruner sobre el aprendizaje, la que promueve el desarrollo de representaciones enactivas y pictóricas que preparan la mente del estudiante para la manipulación de representaciones simbólicas de carácter matemático.

Palabras claves: Enseñanza de la Estadística, pensamiento estadístico, representaciones enactivas, icónicas, simbólicas, Teoría cognitiva de Bruner.

\begin{abstract}
Some situations that arise in the learning of Statistics are analyzed, which could be simplified and used in a better way, if certain dispensable mathematical elements are subtracted and appropriate learning activities are added, which facilitate the familiarization of students with certain fundamental aspects of statistical thinking. Since these proposals use real life experiences, they make possible a cognitive construction based on Bruner's theory of learning, which promotes the development of enactive and pictorial representations that prepare the student's mind for the manipulation of symbolic representations of a mathematical nature.
\end{abstract}

Key words: Statistics teaching, statistical thinking, enactive, iconic, symbolic representations, Bruner's cognitive theory.

\section{LA ESTADÍSTICA DESCRIPTIVA}

$\mathrm{Su}$ objetivo es detectar características de la población que subyacen en los datos de una muestra aleatoria de la población. Desde la perspectiva de la Estadística, hay supuestos que alientan esta búsqueda; en particular, el supuesto de existencia de una distribución de probabilidad para la población que se estudia. Bajo esta perspectiva, el concepto de error aleatorio presente en los datos, es importante ya que se buscan invariantes; es decir, objetos matemáticos que, en alguna forma, están también presentes en los datos observados. 
En la práctica, en el caso de invariantes de una población que son parámetros, es decir, constantes numéricas, existe el riesgo de enseñar a calcular estimaciones de estos parámetros enfatizando la aplicación de una fórmula numérica, en desmedro de otorgar al estudiante una oportunidad de reconocer el error que existe cuando se estima un parámetro por medio de datos muestrales. Este error, siempre presente en cualquier estimación obtenida de los datos de una muestra, se conceptualiza en Estadística, como "error de muestreo" y es un constituyente esencial del pensamiento estadístico. No habiendo objetivo de aprendizaje estadístico, el logro de los objetivos de aprendizaje se asocia a la aplicación correcta de una fórmula matemática.

Mayor peligro aún, si los datos se encuentran agrupados. Se han desarrollado complicados criterios matemáticos para obtener valores aproximados de la moda, la mediana y de los percentiles en general, cuando los datos se encuentran agrupados. Ver, por ejemplo, [8]. Si los datos originales están disponibles, nada más inoficioso que este proceder; hay planillas de cálculo que realizan este trabajo con gran facilidad para el operador. Si la data original está ausente, el retorno a las marcas de clase como valores observados según sea su frecuencia, permitiría una razonable aproximación a los parámetros que se busca estimar.

En resumen, en el caso de la estimación de un invariante numérico, se observa la sobrevaloración del concepto de número en desmedro de la valoración del concepto de error; es decir, la preeminencia del paradigma matemático por sobre el paradigma estadístico. Muy por el contrario, el paradigma estadístico aprecia el parámetro como número teórico, pero asigna gran relevancia a la presencia del error y a la existencia de estimadores que son el constituyente principal de las diferentes vías para calcular, en forma aproximada, estos números teóricos.

Otra cosa ocurre si se busca un invariante de forma de la población, como, por ejemplo, el perfil que toma la curva de la función de distribución de probabilidad. En este caso, la construcción de histogramas a través de una planilla de cálculo es espontánea y facilita la elaboración de representaciones enactivas y también icónicas, que preparan la asimilación de una forma abstracta, de un objeto matemático: la función de distribución de probabilidad. Ver Bruner [1]. Esta forma de proceder sirve como ejemplo a seguir, en el caso de la búsqueda de invariantes numéricos, como se señala a continuación.

Obsérvese que la construcción de histogramas para visualizar la distribución de muestreo de $\hat{\theta}-\theta$; a veces denominado "error de la estimación" (ver Devore [3], página 243), podría ayudar a suplir la carencia de enfoque estadístico en el cálculo de una estimación $\hat{\theta}$ de un parámetro $\theta$ (donde $\theta$ se considera conocido, con fines de aprendizaje). La experiencia de pensamiento estadístico puede profundizarse, variando, por un lado, el estimador que se utiliza y, por otro lado, utilizando muestras 
aleatorias de diferentes tamaños. Esta última variación permitiría visualizar cómo el tamaño de la muestra controla la magnitud del error aleatorio; otro paradigma de la Estadística.

\section{EL CONCEPTO Y LA DEFINICIÓN DE VARIABLE ALEATORIA}

Dado un experimento aleatorio, lo usual es definir el objeto "variable aleatoria" como una función cuyo dominio es el espacio muestral del experimento y cuyo recorrido es un subconjunto de números reales. Esencialmente, esta función convierte los resultados del experimento en números que mantienen la aleatoriedad original del experimento. Es decir, a través de esta transformación, el experimento permite la observación de un número aleatorio. Sin embargo, este objeto matemático función, frecuentemente utilizado para definir el concepto de variable aleatoria, no sólo es prescindible para un apropiado aprendizaje de este concepto; también se ha hipotetizado (ver [2]) que este modo de presentar el concepto de variable aleatoria podría ser fuente de obstáculos en el aprendizaje, los que serían evitables si este concepto se levanta directamente de la realidad como "número aleatorio". Esta doble estrategia de abandonar la definición de variable aleatoria como función matemática y de optar por una definición fenoménica, es decir, que se basa en el reconocimiento de un fenómeno que ocurre en la realidad, podría contribuir sinérgicamente a hacer más efectivos la enseñanza y el aprendizaje de la Estadística.

Como señala Rice (ver [9], página 35), "una variable aleatoria es esencialmente un número aleatorio". Por tanto, si la definición del experimento de interés se extiende hasta incluir la transformación que va del espacio muestral a los números reales y que se utiliza para definir variable aleatoria como función matemática, entonces, los resultados del experimento son números y el experimento en sí adquiere el carácter de número aleatorio susceptible de ser asimilado como variable aleatoria. Procediendo de esta manera, es posible acceder al concepto de variable aleatoria desde el mundo de las experiencias permitiendo su constitución como objeto factible de ser aprehendido directamente del mundo real. Así, una variable aleatoria podría reconocerse en la realidad tal como se reconocen, en la realidad, los números. Utilizando los términos de modelo de primera y segunda generación, introducidos por Gårding, en [4] (ver página 6 y siguientes), podemos decir que al construir el concepto de variable aleatoria como número aleatorio, el objeto variable aleatoria se constituye en un modelo de primera generación. Citando a Guzmán y Hevia [6], “... los modelos de primera generación pueden contribuir de manera importante en el proceso de aprendizaje de las matemáticas, siempre que los objetos representados pertenezcan al ámbito de la experiencia del estudiante". En este caso, los objetos representados son los experimentos aleatorios cuyos resultados son números. La concordancia con la construcción cognitiva de Bruner es evidente; estos experimentos aleatorios permitirán la construcción de representaciones enactivas e icónicas, que potenciarán el aprendizaje significativo del objeto variable aleatoria. 
Hay varios aspectos que se deben destacar; quizás, el más importante, es que un número aleatorio no es un número -la observación de un número aleatorio, sí es un número. Otro aspecto se refiere a la poca utilidad que tiene para el aprendizaje, la definición de variable aleatoria como una función matemática, cuando el experimento aleatorio arroja un número como resultado; como ocurre en el caso del clásico experimento del lanzamiento de un dado. Un tercer aspecto, se refiere a la elección de una distribución de probabilidad para la variable aleatoria de interés. Si se ha procedido a definir una variable aleatoria de interés como una función, entonces, la función de distribución de probabilidad de esta variable aleatoria se haría presente como una segunda función cuyo dominio es el rango de valores que recorre la (función) variable aleatoria. Dado este discurso, pareciera muy posible que la construcción cognitiva que se pretende producir, quede interferida por la presencia cercana de dos objetos matemáticos de la misma naturaleza; las dos funciones aludidas. Esta situación, más que apoyar la construcción del conocimiento, podría dificultarla, desviando el enfoque a aspectos secundarios no relacionados con el objetivo primario de aprendizaje, que son los conceptos de variable aleatoria y de distribución de probabilidad (por ejemplo, la comprensión podría deslizarse hacia el objeto matemático función). Hay estudios que dejan en evidencia la existencia de confusión en los estudiantes en relación a estos dos objetos matemáticos de la misma naturaleza que coexisten en la presentación usual de variable aleatoria. Por otro lado, se ha observado que cuando hay claridad respecto a las diferencias existentes entre estos dos objetos matemáticos, el estudiante no es capaz de reconocer una variable aleatoria presentada como número aleatorio. Ver [2].

\section{EL PROBLEMA QUE CONDUCE AL CONCEPTO DE AJUSTE DE UN MODELO A UNA POBLACIÓN}

El objetivo de la Estadística es obtener información de los datos disponibles, que sea relevante para la situación de interés que se estudia. Etimológicamente, el término "datos" proviene de "datum": lo que es dado; donado a través del acto de conocer, de la intencionalidad de la conciencia. Los datos se organizan por medio de principios explicativos que operan en base a supuestos. En particular, si aceptamos el supuesto de existencia de una distribución de probabilidad para un número aleatorio de interés, con seguridad habrá más de una distribución que se ajuste en forma significativa a los datos observados. Y la elección final siempre será dubitable.

Cada función que satisface los requisitos de una función de distribución de probabilidad podría constituirse en un posible modelo de probabilidad teórico para algún número aleatorio. Por otro lado, la asignación teórica de probabilidad bajo ciertas condiciones experimentales suficientemente específicas, inevitablemente conduce a una única distribución de probabilidad teórica (considérese, por ejemplo, la 
distribución binomial, la distribución hipergeométrica, la distribución de Poisson, etc.) lo que refuerza la creencia en la búsqueda de una única e ideal distribución de probabilidad para los datos que se estudian. De aquí, la importancia de distinguir entre una variable aleatoria cuya distribución de probabilidad, aunque existente, es desconocida y una variable aleatoria teórica cuya distribución de probabilidad es conocida con absoluta certeza. Esto explica la necesidad que se tiene en el pensamiento estadístico de mantener representaciones independientes para la variable aleatoria y para su posible distribución de probabilidad. Dado un número aleatorio que se quiere estudiar, la variable aleatoria es única; sin embargo, la distribución de probabilidad podría estar lejos de ser determinada y cualquier distribución de probabilidad que se ajuste razonablemente a los datos observados será de valor para el estudio.

A diferencia de lo señalado anteriormente, es frecuente encontrar en la literatura, listados preeminentes y exhaustivos de estos modelos teóricos junto a una ausencia de reconocimiento explícito a la exigencia cognitiva, propia del pensamiento estadístico, de mantener autonomía entre la variable aleatoria y su distribución de probabilidad, la que, siendo desconocida, podría quedar aproximada por varios modelos. Esta simplificación teórica que se presenta frecuentemente en la literatura podría ser atribuible a un enfoque eminentemente teoricista (ver Gascon [5]) que minimiza el rol que tienen en el aprendizaje de la Estadística los números aleatorios observables en la realidad y que, usualmente, no tienen una distribución de probabilidad conocida con certeza. Estos números aleatorios, por emerger de la realidad, exigen de una representación autónoma como variables aleatorias, independientemente del grado de definición que adquieran sus distribuciones aproximadas, en caso que existan.

\section{EL TEOREMA DE BAYES}

Otra simplificación teoricista, aparece en la enseñanza y aprendizaje del Teorema de Bayes. El recálculo de las probabilidades a priori, tiene valor práctico en la medida que hay nueva información que se agrega; por ejemplo, a través de una muestra ad hoc. Si la estructura de probabilidades es conocida a priori en su totalidad, poca o ninguna información agrega este teorema a lo que ya se conoce; más allá de reconocer las relaciones teóricas existentes entre las diferentes probabilidades en juego. Precisamente, la riqueza de aplicaciones de este teorema proviene de la inexistencia de conocimiento acabado de la estructura de probabilidades que es de interés y de su cruce con otras fuentes de información; usualmente, a través de la estimación por otros medios de las probabilidades condicionales denominadas "verosimilitudes". 


\section{LA DEFINICIÓN DE EVENTO}

Otro obstáculo que tiene su origen en la predominancia del paradigma axiomático (ver [5]), toma lugar en la definición de eventos de un espacio muestral (finito). Dado un experimento $\mathcal{E}$, resulta matemáticamente simple definir los eventos (o sucesos) como subconjuntos del espacio muestral $S$ del experimento $\mathcal{E}$; y así se procede en la práctica, en la totalidad de la literatura disponible. Pero esto implica que los eventos se presentan como modelos de segunda generación, lo que inmediatamente aleja este concepto del campo de experiencias del estudiante. Sin embargo, existe una manera simple de definir eventos a través del lenguaje natural, si uno entiende que estos eventos simplemente describen propiedades que podrían tener los resultados del experimento que se estudia (ver [7]). Si un evento se define como un atributo, característica o propiedad que podrían tener algunos resultados del experimento $\mathcal{E}$, entonces, no sólo nos estamos refiriendo a fenómenos que ocurren en la realidad misma y que, por tanto, permiten la formación de representaciones enactivas e icónicas, sino que, también, a través de cada una de estas características estamos definiendo naturalmente un subconjunto del espacio muestral, cuando esta característica se considera como una especificación para los elementos del espacio muestral. Esto permite lograr la concepción de evento como conjunto, en un paso subsecuente. Presentados los eventos a través de estos dos pasos, se inducen representaciones tanto enactivas como icónicas que conviven con las simbólicas otorgando significado a los objetos matemáticos que representan a los eventos; es decir, a los subconjuntos del espacio muestral.

A diferencia de los puntos anteriores, en los dos puntos que siguen, se destaca la necesidad de un mayor enfoque matemático en la situación de enseñanza y aprendizaje de la Estadística que se indica.

\section{LA (DESCONOCIDA) DENSIDAD NORMAL}

Estudiemos la siguiente ecuación que define una distribución de probabilidad normal $N\left(\mu ; \sigma^{2}\right)$, con media $\mu$ y varianza $\sigma^{2}$.

$$
f(x)=\frac{1}{\sqrt{2 \pi} \sigma} e^{-\frac{(x-\mu)^{2}}{2 \sigma^{2}}}
$$

Observe que el punto estacionario de la campana de Gauss se encuentra en $x=\mu$; por tanto, la altura de la campana es igual a $\frac{1}{\sqrt{2 \pi} \sigma} \approx \frac{0,4}{\sigma}$. Dado que aproximadamente $100 \%$ del área bajo la curva se encuentra en el intervalo $[\mu-3 \sigma ; \mu+3 \sigma]$, se concluye que la parte principal de la campana bajo la cual hay área significativa se encuentra, prácticamente en su totalidad, en el interior de un rectángulo cuya base tiene longitud $6 \sigma$ y altura igual a $\frac{0,4}{\sigma}$; estando la base de este rectángulo centrada en $\mu$. Este 
rectángulo, ha sido denominado "rectángulo de la forma de la normal" en [7]. (En particular, la parte principal de la curva de la distribución normal estandarizada bajo la cual hay área significativa, se encuentra en un rectángulo cuya base tiene longitud 6 y altura 0,4 .)

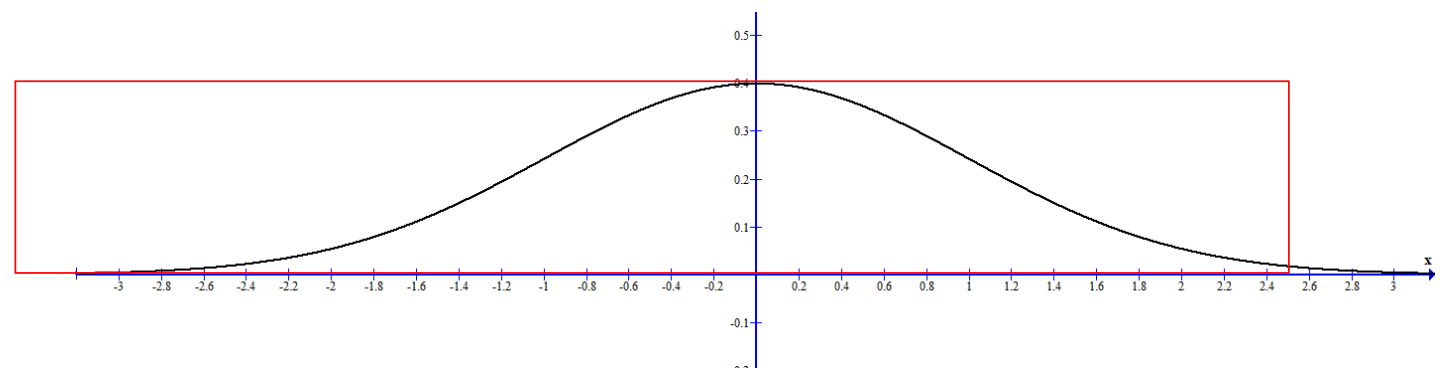

Recordemos que si la población se distribuye normal $N\left(\mu ; \sigma^{2}\right)$, la distribución de muestreo de los promedios de muestras independientes de tamaño $n$ se distribuye normal $N\left(\mu ; \frac{\sigma^{2}}{n}\right)$, con desviación estándar $\frac{\sigma}{\sqrt{n}}$. Esta última curva normal se encuentra en un rectángulo de forma cuya base tiene como punto medio el parámetro $\mu$, posiblemente desconocido. Observe que si el tamaño $n$ de la muestra es grande, entonces la base de este rectángulo cuya longitud es $6 \frac{\sigma}{\sqrt{n}}$ tiende a 0 y, por tanto, cualquier observación del promedio muestral para este tamaño de muestra, acertará razonablemente en estimar el promedio desconocido $\mu$.

Lo anterior, podría anticipar o confirmar, en un registro visual, la denominada Ley de los Grandes Números y la noción más sofisticada de convergencia en probabilidad de un estimador al parámetro que se estima.

\section{LAS TABLAS NORMALES Y LA OMISIÓN DE LA FUNCIÓN DE DISTRIBUCIÓN DE PROBABILIDAD ACUMULATIVA $\Phi(z)$}

La omisión de la función de distribución de probabilidad acumulativa $\Phi(z)$ de la distribución normal estandarizada en el cálculo de probabilidades normales produce un punto ciego, una ausencia de comprensión en la mente del estudiante, dentro de la construcción del pensamiento estadístico. Se enseña a operar con estas tablas desconociendo la existencia de objeto matemático $\Phi(z)$ cuyo realce permitiría completar, al menos en este aspecto, la construcción de pensamiento estadístico coherente.

El valor de las distribuciones acumulativas se hace innegable cuando se enfrenta el problema de la generación de números aleatorios que siguen una distribución de probabilidad dada. Sabemos que bajo condiciones simples de establecer, la distribución de probabilidad acumulativa es invertible lo que hace posible la generación de números aleatorios cuya distribución de probabilidad es la dada. Ejemplos de este tipo son absolutamente posibles de ser utilizados en los años terminales de la enseñanza media, 
previos a la enseñanza superior. Nuevamente, la producción de observaciones de un cierto número aleatorio, simuladas por medio de la manipulación de software en un procesador, permitiría consolidar el concepto de variable aleatoria por medio de la formación de representaciones enactivas e icónicas.

\section{REFERENCIAS BIBLIOGRÁFICAS}

[1] Bruner, Jerome S. (2006): The course of cognitive growth, en In Search of Pedagogy, Volume I. Routledge,

[2] Contreras, Leny (2017): Dificultades en la comprensión del concepto de Variable Aleatoria. Trabajo final para optar al grado de Magíster en Didáctica de la Matemática, Facultad de Educación, Universidad Alberto Hurtado.

[3] Devore, Jay L. (2012): Probabilidad y estadística para ingeniería y ciencias, octava edición. Cengage Learning.

[4] Gårding, Lars (1977): Encounters with Mathematics. Springer-Verlag.

[5] Gascón, Josep (2001): Incidencia del modelo epistemológico de las matemáticas sobre el modelo docente. RELIME, Vol.4 Num. 2, 129-159.

[6] Guzmán, Ismenia y Hevia, Héctor (2008): Modelos Matemáticos y su Incidencia en el Aprendizaje de las Matemáticas. Informe Técnico CIDIC, Universidad Adolfo Ibáñez. No ha sido publicado.

[7] Hevia, Héctor (2013): Didáctica Fenomenológica de la Modelación Estadística de los Datos; micro curso dictado en el Primer Congreso Internacional de Estadística, Universidad Nacional de Trujillo, Perú.

[8] Martín-Pliego, Francisco J. (2004): Introducción a la Estadística Económica y Empresarial, tercera edición. Thomson.

[9] Rice, John A. (2007): Mathematical Statistics and Data Analysis, third edition. Thomson. 